\title{
NOTE ON THE TENSOR PRODUCT OF BANACH ALGEBRAS ${ }^{1}$
}

\author{
BERNARD R. GELBAUM
}

0. Introduction. In [2] the maximal ideal space of the tensor product of two commutative Banach algebras was studied. One of the results was: Let $A_{3}=A_{1} \otimes_{0} A_{2}$ be the "greatest cross-norm" $[3 ; 4]$ tensor product of two commutative Banach algebras $A_{1}$ and $A_{2}$. Let $\mathfrak{M}_{1}, \mathfrak{M}_{2}$, $\mathfrak{M}_{3}$ be the corresponding spaces of regular maximal ideals. Then $\mathfrak{M}_{3}$ and $\mathfrak{M}_{1} \times \mathfrak{M}_{2}$ are "naturally" homeomorphic if the weak* topologies are used in all spaces. In the following, we extend the discussion to the case in which no commutativity is assumed. ${ }^{2}$

1. Tensor products. Let $A_{1}$ and $A_{2}$ be Banach algebras and let $C$ be the complex number system. We consider [3] a subset $F$ of $C^{A_{1} \times A_{2}}$ :

$$
\begin{aligned}
F=\left\{f \mid f \in C^{A_{1} \times A_{2}}, f\left(0, x_{2}\right)=f\left(x_{1}, 0\right)\right. & =0, \\
& \left.\sum_{\left(x_{1}, x_{2}\right)}\left|f\left(x_{1}, x_{2}\right)\right|\left\|x_{1}\right\|_{1}\left\|x_{2}\right\|_{2}<\infty\right\},
\end{aligned}
$$

where $x_{i} \in A_{i}, i=1,2$. Since each $f$ in $F$ is nonzero on a set that is countable or finite, to each $f$ there corresponds a sequence (finite or infinite) $\left\{\left(x_{11}, x_{21}\right),\left(x_{12}, x_{22}\right), \cdots\right\}$ consisting of just the pairs $\left(x_{1}, x_{2}\right)$ where $f\left(x_{1}, x_{2}\right) \neq 0$. Addition of elements of $f$ is defined by addition of functions. Multiplication is defined via a form of convolution: If $f, g \in F, f * g=h$ is defined by:

$$
h\left(0, x_{2}\right)=h\left(x_{1}, 0\right)=0, \quad h\left(x_{1}, x_{2}\right)=\sum_{h_{1} b_{1}=x_{1} ; a_{2} b_{2}=x_{2}} f\left(a_{1}, a_{2}\right) g\left(b_{1}, b_{2}\right)
$$

if $x_{1}, x_{2} \neq 0$. We note:

Presented to the Society September 4, 1959; received by the editors December 26, 1959 and, in revised form, September 3, 1960 and May 4, 1961.

1 This research was supported in part by the United States Air Force through Air Force Office of Scientific Research of the Air Research and Development Command, under contract No. AF 49 (638)-526, and in part by the National Science Foundation under grant NSF-G11048. The author thanks these organizations for their assistance. Reproduction of the paper in whole or in part is permitted for any purposes of the United States Government.

2 The author is much indebted to the referee of an earlier draft of this research. At his suggestion, arguments that were too compressed for intelligible reading have been elaborated. 


$$
\begin{aligned}
& \sum_{\left(x_{1}, x_{2}\right)}\left|h\left(x_{1}, x_{2}\right)\right|\left\|x_{1}\right\|_{1}\left\|x_{2}\right\|_{2} \\
& \quad \leqq \sum_{\left(a_{1}, a_{2}\right),\left(b_{1}, b_{2}\right)}\left|f\left(a_{1}, a_{2}\right)\right|\left|g\left(b_{1}, b_{2}\right)\right|\left\|a_{1}\right\| 1\left\|b_{1}\right\|{ }_{1}\left\|a_{2}\right\|_{2}\left\|b_{2}\right\|_{2} \\
& =\left(\sum_{\left(a_{1}, a_{2}\right)}\left|f\left(a_{1}, a_{2}\right)\right|\left\|a_{1}\right\|_{1}\left\|a_{2}\right\|_{2}\right)\left(\sum_{\left(b_{1}, b_{2}\right)} g\left(b_{1}, b_{2}\right)\left\|b_{1}\right\|_{1}\left\|b_{2}\right\|_{2}\right)<\infty .
\end{aligned}
$$

Thus $h \in F$ and the value of $h\left(x_{1}, x_{2}\right)$ is given by an absolutely convergent series. Relative to the operations defined, $F$ is an algebra over $C$.

If $\|f\|_{3}^{\prime}$ is taken as $\sum_{\left(x_{1}, x_{2}\right)}\left|f\left(x_{1}, x_{2}\right)\right|\left\|x_{1}\right\|_{1}\left\|x_{2}\right\|_{2}$, then an analog of the proof that $l_{1}$ is a Banach space shows that $F$ is a Banach space. The relation $\|f * g\|_{3}^{\prime} \leqq\|f\|_{3}^{\prime}\|g\|_{3}^{\prime}$ follows from the computation of the preceding paragraph. Hence $F$ is a Banach algebra.

In $F$ let $I$ be the closed ideal generated by all functions $f$ of one of the following forms $\left(x_{1}, x_{1}^{\prime}, x_{2}, x_{2}^{\prime}\right.$ are arbitrary, $\alpha$ is a complex number):

(i) $f\left(x_{1}, x_{2}\right)=f\left(x_{1}, x_{2}^{\prime}\right)=-f\left(x_{1}, x_{2}+x_{2}^{\prime}\right), f=0$ otherwise;

(ii) $f\left(x_{1}, x_{2}\right)=f\left(x_{1}^{\prime}, x_{2}\right)=-f\left(x_{1}+x_{1}^{\prime}, x_{2}\right), f=0$ otherwise;

(iii) $f\left(x_{1}, x_{2}\right)=-\alpha f\left(\alpha x_{1}, x_{2}\right), f=0$ otherwise;

(iv) $f\left(x_{1}, x_{2}\right)=-\alpha f\left(x_{1}, \alpha x_{2}\right), f=0$ otherwise.

We assert that when $F / I$ is given its quotient space norm it is isometrically isomorphic to $A_{3}$, the greatest cross-norm tensor product $A_{1} \otimes_{g} A_{2}$ of $A_{1}$ and $A_{2}$. The argument rests on the following statements each of which is simply proved.

(a) In $F$, the set $F_{0}$ of functions $f$ that are nonzero on sets that are at most finite is dense in $F$.

(b) $F_{0}$ is a normed algebra containing the generators of $I$. Let $I_{0}$ be the closed ideal generated in $F_{0}$ by the generators of $I$. Then $I_{0}$ is a linear space in $F_{0}$ and $I_{0}$ is dense in $I$.

(c) For $f$ in $F_{0}$, the quotient norm of $f / I_{0}$ is precisely the greatest cross-norm of the equivalence class represented by $f$.

(d) The mapping $\phi_{0}: F_{0} / I_{0} \rightarrow A_{3}$ defined by: $\phi_{0}\left(f / I_{0}\right)=$ equivalence class represented by $f$ is an isometry onto a dense subset of $A_{3}$. The extension $\phi$ of $\phi_{0}$ to $F / I$ is an isometry of $F / I$ onto $A_{3}$.

In dealing with $A_{3}$ (where the norm will be denoted by $\|\cdots\|_{3}$ ) we shall often work with representatives of elements in $F / I$. For any $f \in F$, the element $g$ given by: $g\left(x_{1}, x_{2}\right)=1$ if $\left(x_{1}, x_{2}\right)=\left(f\left(x_{1}^{\prime}, x_{2}\right) x_{1}^{\prime}, x_{2}\right)$ and $g=0$ otherwise, satisfies the equation $f / I=g / I$. Thus, ultimately an element of $A_{3}$ will be symbolized by a formal sum $\sum_{n=1}^{\infty}\left(x_{1 n} \otimes x_{2 n}\right)$ where $\sum_{n=1}^{\infty}\left\|x_{1 n}\right\|_{1}\left\|x_{2 n}\right\|_{2}<\infty$. Very often we shall omit reference to representatives. For example, we shall say simply "the element 


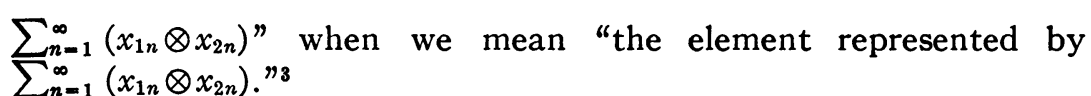

2. $\mathfrak{M}_{1} \times \mathfrak{M}_{2} \rightarrow \mathfrak{M}_{3}$. Let $\left(M_{1}, M_{2}\right)$ be in $\mathfrak{M}_{1} \times \mathfrak{M}_{2}$. Define the homomorphism $E_{3}: A_{3} \rightarrow\left(A_{1} / M_{1}\right) \otimes_{0}\left(A_{2} / M_{2}\right)$ by the formula: $E_{3}\left(\sum_{n=1}^{\infty}\left(x_{1 n} \otimes x_{2 n}\right)\right)=\sum_{n=1}^{\infty}\left(E_{1}\left(x_{1 n}\right) \otimes E_{2}\left(x_{2 n}\right)\right)$, where $E_{1}$ and $E_{2}$ are the canonical epimorphisms $E_{i}: A_{i} \rightarrow A_{i} / M_{i}, i=1,2$. Let $\|\cdots\|_{\mathrm{I}}$, $\|\ldots\|_{\text {II }}$ denote the quotient norms in $A_{1} / M_{1}$ and $A_{2} / M_{2}$. Since these algebras are nontrivial so is $\left(A_{1} / M_{1}\right) \otimes_{\theta}\left(A_{2} / M_{2}\right)$ nontrivial. Thus $E_{3}^{-1}(0)=M_{3}$ is a proper ideal in $A_{3}$. If $u_{1}$ and $u_{2}$ are identities modulo $M_{1}$ and $M_{2}$ then $u=u_{1} \otimes u_{2}$ is an identity modulo $M_{3}$. Thus $M_{3}$ is regular. Since the norm $\|\cdots\|_{\text {IIr }}$ in $E_{3}\left(A_{3}\right)$ satisfies

$$
\begin{aligned}
\| E_{3}\left(\sum_{n=1}^{\infty}\left(x_{1 n} \otimes x_{2 n}\right) \|_{\mathrm{III}}\right. & \leqq \sum_{n=1}^{\infty}\left\|E_{1}\left(x_{1 n}\right)\right\|_{\mathrm{I}}\left\|E_{2}\left(x_{2 n}\right)\right\|_{\mathrm{II}} \\
& \leqq \sum_{n=1}^{\infty}\left\|x_{1 n}\right\|_{1}\left\|x_{2 n}\right\|_{2}
\end{aligned}
$$

we see that $E_{3}$ is norm-decreasing and hence that $E_{3}$ is continuous. Hence $M_{3}$ is closed.

If $M_{3}$ is not maximal, let $M_{3} \subset N_{3}$, a regular maximal ideal. Let $F_{3}: A_{3} \rightarrow A_{3} / N_{3}$ be the canonical epimorphism and define $G_{1}$ and $G_{2}$ by the formulas

$$
\begin{aligned}
& G_{1}\left(x_{1}\right)=E_{3}\left(u x_{1}\right), \\
& G_{2}\left(x_{2}\right)=E_{3}\left(x_{2} u\right) .
\end{aligned}
$$

Then $G_{i}\left(A_{i}\right) \subset A_{3} / M_{3}, i=1,2$. We prove now

Lemma 1. A regular ideal $I_{3}$ in $A_{3}$ is a right and left $A_{1}$ - and $A_{2^{-}}$ ideal.

Proof. Let $u$ be an identity modulo $I_{3}$ in $A_{3}, x_{1} \in A_{1}, v \in I_{3}$. Then $u x_{1} v-x_{1} v \in I_{3}$ and thus $u x_{1} v / I_{3}-x_{1} v / I_{3}=0$. But $u x_{1} v / I_{3}=\left(u x_{1} / I_{3}\right)\left(v / I_{3}\right)$ $=0$. Thus $x_{1} v \in I_{3}$. Similarly $v x_{1} \in I_{3}$, and $x_{2} v, v x_{2} \in I_{3}$ for $x_{2} \in A_{2}$.

From Lemma 1 we see that $G_{2}\left(x_{2} y_{2}\right)=E_{3}\left(x_{2} y_{2} u\right)=E_{3}\left(x_{2}\left(m_{3}+u y_{2} u\right)\right)$ $=E_{3}\left(x_{2} u y_{2} u\right)=G_{2}\left(x_{2}\right) G_{2}\left(y_{2}\right) \quad$ (where $\left.m_{3} \in M_{3}\right)$. Note also: $\left\|G_{2}\left(x_{1}\right)\right\|_{\text {III }}$ $=\left\|E_{3}\left(u x_{1}\right)\right\|_{\mathrm{III}} \leqq\left\|u x_{1}\right\|_{3} \leqq k\left\|x_{1}\right\|_{1}$. It is clear now that $G_{2}$ (and similarly $\left.G_{1}\right)$ is a continuous homomorphism.

If $\left(x_{1}, x_{2}\right) \in M_{1} \times M_{2}$ then $u x_{1}=\left(u_{1} x_{1} \otimes u_{2}\right)=w$ is such that $E_{3}(w)=0$, i.e., $G_{1}\left(x_{1}\right)=0$. Thus $M_{1} \subset G_{1}^{-1}(0)$. Similarly, $M_{2} \subset G_{2}^{-1}(0)$. Hence either $M_{i}=G_{i}^{-1}(0)$ or $G_{i}\left(A_{i}\right)=0, i=1$, 2. Since $G_{i}\left(u_{i}\right) \neq 0$, we see

${ }^{8} A_{8}$ may be regarded as a left and right $A_{1-}$ and $A_{2}$-module, e.g., $x_{1}\left(y_{1} \otimes y_{2}\right)$ $=\left(x_{1} y_{1} \otimes y_{2}\right)$, where $x_{1}, y_{1} \in A_{1}, y_{2} \in A_{2}$. 
$G_{i}^{-1}(0)=M_{i}$. If $H_{i}$ are engendered by $F_{3}$ as $G_{i}$ are engendered by $E_{3}$ we find [2] $M_{i}=H_{i}^{-1}(0), i=1,2$. Following the argument in [2], we obtain $H_{i}=\alpha_{i} G_{i}=\beta_{i} E_{i}$ where $\alpha_{i}, \beta_{i}$ are isometric $C$-automorphisms of $A_{i} / M_{i}$. Finally $E_{3}=\left(\beta_{1} \otimes \beta_{2}\right)^{-1} F_{3}$, whence $E_{3}\left(A_{3}\right)$ is simple and $M_{3}$ is maximal.

We remark that $E_{3}$ is an epimorphism. Indeed, if

$$
\sum_{n=1}^{\infty}\left(E_{1}\left(x_{1 n}\right) \otimes E_{2}\left(x_{2 n}\right)\right)=w \in\left(A_{1} / M_{1}\right) \otimes_{g}\left(A_{2} / M_{2}\right),
$$

we can choose $\left(y_{1 n}, y_{2 n}\right) \in A_{1} \times A_{2}$ so that $E_{i}\left(y_{i n}\right)=E_{i}\left(x_{i n}\right), i=1,2$, $n=1,2, \cdots$, and such that $\left\|y_{1 n}\right\|_{1}<\left\|E_{1}\left(x_{1 n}\right)\right\|_{\mathrm{I}}+2^{-n},\left\|y_{2 n}\right\|_{2}$ $<\left\|E_{2}\left(x_{2 n}\right)\right\|_{\mathrm{II}}+2^{-n}$. (We may and do assume $\left\|E_{1}\left(x_{1 n}\right)\right\|_{\mathrm{I}}=\left\|E_{2}\left(x_{2 n}\right)\right\|_{\mathrm{II}}$. For, in each nontrivial term we may use the equivalent representative $\alpha E_{1}\left(x_{1 n}\right) \otimes \alpha^{-1} E_{2}\left(x_{2 n}\right)$ where $\alpha=+\left[\left(\left\|E_{1}\left(x_{1 n}\right)\right\|_{1}\right)\left(\left\|E_{2}\left(x_{2 n}\right)\right\|_{1 \mathrm{II}}\right)^{-1}\right]^{1 / 2}$. $)$ Then if $z=\sum_{n=1}^{\infty}\left(y_{1 n} \otimes y_{2 n}\right)$ we see $E_{3}(z)=w$.

We note (for later use) that $G_{1}\left(A_{1}\right)$ and $G_{2}\left(A_{2}\right)$ are commuting subalgebras of $A_{3} / M_{3}$. For

$$
\begin{aligned}
& G_{1}\left(x_{1}\right) G_{2}\left(x_{2}\right)=E_{3}\left(u x_{1}\right) E_{3}\left(x_{2} u\right)=E_{3}\left(u_{1} x_{1} \otimes u_{2}\right) E_{3}\left(u_{1} \otimes x_{2} u_{2}\right) \\
& =\left(E_{1}\left(u_{1} x_{1}\right) \otimes E_{2}\left(u_{2}\right)\right) \cdot\left(E_{1}\left(u_{1}\right) \otimes E_{2}\left(x_{2} u_{2}\right)\right)=E_{1}\left(x_{1}\right) \otimes E_{2}\left(x_{2}\right) \\
& =\left(E_{1}\left(u_{1}\right) \otimes E_{2}\left(x_{2} u_{2}\right)\right) \cdot\left(E_{1}\left(u_{1} x_{1}\right) \otimes E_{2}\left(u_{2}\right)\right)=E_{3}\left(x_{2} u\right) E_{3}\left(u x_{1}\right) \\
& \quad=G_{2}\left(x_{2}\right) G_{1}\left(x_{1}\right) .
\end{aligned}
$$

Hence [1] there is a homomorphism $T: G_{1}\left(A_{1}\right) \otimes_{0} G_{2}\left(A_{2}\right) \rightarrow A_{3} / M_{3}$ given by $T\left(\sum_{n=1}^{\infty}\left(G_{1}\left(x_{1 n}\right) \otimes G_{2}\left(x_{2 n}\right)\right)\right)=\sum_{n=1}^{\infty} G_{1}\left(x_{1 n}\right) G_{2}\left(x_{2 n}\right)$. Since $T\left(G_{1}\left(u_{1}\right) \otimes G_{2}\left(u_{2}\right)\right)=$ the identity of $A_{3} / M_{3}$, and since $G_{1}\left(A_{1}\right) \otimes_{g} G_{2}\left(A_{2}\right)$ is simple we see $T^{-1}(0)=0$, i.e., $T$ is a monomorphism. From our definitions it now follows that $T$ is an isomorphism.

Similarly, if $\gamma_{i}$ are isometric isomorphisms $\gamma_{i}: G_{i}\left(A_{i}\right) \rightarrow B_{i}, i=1,2$, there is an isomorphism $T^{\prime}: B_{1} \otimes_{g} B_{2} \rightarrow A_{3} / M_{3}$ given by

$$
T^{\prime}\left(\sum_{n=1}^{\infty}\left(y_{1 n} \otimes y_{2 n}\right)\right)=T\left(\sum_{n=1}^{\infty}\left(\gamma_{1}^{-1}\left(y_{1 n}\right) \otimes \gamma_{2}^{-1}\left(y_{2 n}\right)\right)\right) \text {. }
$$

Finally, the association between pairs $\left(M_{1}, M_{2}\right)$ and ideals $M_{3}$ is 1-1. For if $\left(M_{1}, M_{2}\right) \neq\left(M_{1}^{\prime}, M_{2}^{\prime}\right)$, e.g., if $M_{1} \neq M_{1}^{\prime}$, choose $x_{1} \in M_{1} \backslash M_{1}^{\prime}$ and $x_{2} \in M_{2} \backslash M_{2}^{\prime}$ if $M_{2} \neq M_{2}^{\prime}$ (otherwise choose $x_{2} \notin M_{2}$ ). Then $E_{3}\left(x_{1} \otimes x_{2}\right)=0$, and $E_{3}^{\prime}\left(x_{1} \otimes x_{2}\right) \neq 0$, i.e., $\left(M_{1}, M_{2}\right)$ and $\left(M_{1}^{\prime}, M_{2}^{\prime}\right)$ engender distinct epimorphisms $E_{3}$ and $E_{3}^{\prime}$.

3. $\mathfrak{M}_{3} \rightarrow \mathfrak{M}_{1} \times \mathfrak{M}_{2}$. Consider the algebras $A_{i e_{i}}$ obtained by adjoining (if necessary) identities $e_{i}$ to $A_{i}, i=1,2$. It is to be noted that $A_{4}=A_{1_{e_{1}}} \otimes_{0} A_{2 \varepsilon_{2}}$ is in general not the same as $A_{3 e_{3}}$, the result of 
adjoining (if necessary) an identity $e_{3}$ to $A_{3}$. A typical element $z$ of $A_{4}$ may be represented by $\alpha\left(e_{1} \otimes e_{2}\right)+e_{1} \otimes x_{2}+x_{1} \otimes e_{2}+\sum_{n=1}^{\infty}\left(x_{1 n} \otimes x_{2 n}\right)$ where $x_{1}, x_{1 n} \in A_{1}, x_{2}, x_{2 n} \in A_{2}, \alpha \in C, \sum_{n=1}^{\infty}\left\|x_{1 n}\right\| 1\left\|x_{2 n}\right\|_{2}<\infty$. There is an obvious isometric isomorphic copy of $A_{3}$ in $A_{4}$. We denote the copy of $A_{3}$ by $A_{3}$.

Lemma 2. $A_{3}$ is an ideal in $A_{4}$.

Proof. Let $z$ be as above, and let $w=\sum_{n=1}^{\infty}\left(w_{1 n} \otimes w_{2 n}\right) \in A_{3}$. Then $z w=\alpha w+w x_{2}+x_{1} w+v w$ where $v=\sum_{n=1}^{\infty}\left(x_{1 n} \otimes x_{2 n}\right)$. Since all four summands of $z w$ are in $A_{3}$ the result follows.

A similar argument employing Lemma 1 shows the following.

Lemma 3. $A$ closed ideal $I_{3}$ of $A_{3}$ is a closed ideal in $A_{4}$.

Lemma 4. Let $M_{3}$ be a regular maximal ideal of $A_{3}, u$ an identity (in $A_{3}$ ) modulo $M_{3}: u A_{3}-A_{3}, A_{3} u-A_{3} \subset M_{3}$. In $A_{4}$ let $M_{4}^{0}=\left\{z \mid u z, z u \in M_{3}\right\}, A_{4}^{0}=\left[x_{1} \otimes e_{2}+e_{1} \otimes x_{2}\right] \oplus A_{3}$. Then $M_{4}^{0}$ is a (regular) ideal in $A_{4}, u$ is an identity (in $A_{4}$ ) modulo $M_{4}^{0}$ and $M_{4}^{0} \mp A_{4}^{0}$.

Proof. For $z \in M_{4}^{0}, w \in A_{4}$, we see in succession: $u z, u z w, u z w u$, $u(z w u)-z w u, z w u \in M_{3}$. Hence $M_{4}^{0}$ is a right ideal and by a similar proof is a left ideal.

Writing $e_{4}=e_{1} \otimes e_{2}$, we see $u\left(u-e_{4}\right)=u^{2}-u \in M_{3}$, whence $u-e_{4}$ $\in M_{4}^{0} \backslash A_{4}^{0}$.

Since (Lemma 2) $A_{3}$ is an ideal in $A_{4}$, we find that if $z \in A_{4}$ then $u z, \quad z u \in A_{3}$ and $(u z-z) u=u(z u)-z u, u(u z-z)=u(u z)-u z \in M_{3}$, whence $u z-z \in M_{4}^{0}$.

We now consider several cases:

Case I. Assume $A_{1}$ and $A_{2}$ have identities. Define mappings $F_{i}$ : $A_{i} \rightarrow A_{3} / M_{3}, i=1,2$ by the (symbolically given) formulas $F_{1}\left(A_{1}\right)$ $=\left(A_{1} \otimes e_{2}\right) / M_{3}, F_{2}\left(A_{2}\right)=\left(e_{1} \otimes A_{2}\right) / M_{3}$. Then $F_{i}^{-1}(0)$ are regular ideals $N_{i}$ of $A_{i}, i=1,2$.

We find the pair associated with $M_{3}$ by treating several subcases.

Case 1. $N_{1}$ and $N_{2}$ are both maximal. Then as in $\$ 2$, let them engender an $N_{3} \in \mathfrak{M}_{3}$ and let $G_{3}$ be the canonical epimorphism $G_{3}$ : $A_{3} \rightarrow\left(A_{1} / N_{1}\right) \otimes_{g}\left(A_{2} / N_{2}\right)$. We shall show $N_{3} \subset M_{3}$ (whence we shall have shown $N_{3}=M_{3}$ since both are maximal ideals). Thus let $z=\sum_{n=1}^{\infty}\left(x_{1 n} \otimes x_{2 n}\right) \in A_{3}$ and assume $G_{3}(z)=0$. Then

$$
\sum_{n=1}^{\infty}\left(F_{1}\left(x_{1 n}\right) \otimes F_{2}\left(x_{2 n}\right)\right)=0 .
$$

$F_{1}\left(A_{1}\right)$ and $F_{2}\left(A_{2}\right)$ are commuting subalgebras of $A_{3} / M_{3}$ and thus $T:\left(A_{1} / N_{1}\right) \otimes_{g}\left(A_{2} / N_{2}\right) \rightarrow A_{3} / M_{3}$, given by $T\left(\sum_{n=1}^{\infty}\left(F_{1}\left(x_{1 n}\right) \otimes F_{2}\left(x_{2 n}\right)\right)\right)$ 
$=\sum_{n=1}^{\infty} F_{1}\left(x_{1 n}\right) F_{2}\left(x_{2 n}\right)$, is a homomorphism. (Since $T\left(F_{1}\left(u_{1}\right) \otimes F_{2}\left(u_{2}\right)\right.$ ) $=$ the identity of $A_{3} / M_{3}$, and since $F_{1}\left(A_{1}\right) \otimes_{0} F_{2}\left(A_{2}\right)$ is simple, $T$ is a monomorphism.) Hence

$F_{3}(z)=\sum_{n=1}^{\infty}\left(F_{1}\left(x_{1 n}\right) F_{2}\left(x_{2 n}\right)\right)=T\left(\sum_{n=1}^{\infty}\left(F_{1}\left(x_{1 n}\right) \otimes F_{2}\left(x_{2 n}\right)\right)\right)=T(0)=0$.

Thus $N_{3} \subset M_{3}$.

Case 2. $N_{1}$ is not maximal; $N_{2}$ is maximal. Let $N_{1} \subset M_{1} \in \mathfrak{M}_{1}$. Then $M_{1}$ and $N_{2}$ engender an $M_{3}^{\prime} \in \mathfrak{M}_{3}$. We shall show $M_{3}^{\prime} \subset M_{3}$ (whence, again, we shall have shown $M_{3}^{\prime}=M_{3}$ ). This time let $G_{3}^{\prime}$ be the canonical epimorphism $G_{3}^{\prime}: A_{3} \rightarrow\left(A_{1} / M_{1}\right) \otimes_{g}\left(A_{2} / N_{2}\right)$, and let $G_{1}^{\prime}, G_{2}^{\prime}$ arise from $G_{3}^{\prime}$ according to the procedure in $\S 2$. Then $\alpha_{2} G_{2}^{\prime}=F_{2}$, where $\alpha_{2}$ is an isometric $C$-automorphism of $F_{2}\left(A_{2}\right)$. As indicated in $\S 2$, there is an isomorphism $T: G_{1}^{\prime}\left(A_{1}\right) \otimes_{0} G_{2}^{\prime}\left(A_{2}\right) \rightarrow A_{3} / M_{3}^{\prime}$. If $L: A_{1} / N_{1} \rightarrow\left(A_{1} / N_{1}\right) /\left(F_{1}\left(M_{1}\right)\right)$ is the canonical epimorphism, then by virtue of the "second isomorphism theorem" $L F_{1}=\alpha_{1} G_{1}^{\prime}$, where $\alpha_{1}$ is an isometric $C$-automorphism of $G_{1}^{\prime}\left(A_{1}\right)$. If $G_{3}(z)=0$, then $\sum_{n=1}^{\infty}\left(E_{1}\left(x_{1 n}\right) \otimes F_{2}\left(x_{2 n}\right)\right)=0,\left(E_{1}: A_{1} \rightarrow A_{1} / M_{1}\right)$. Via $C$-automorphisms we can conclude $\sum_{n=1}^{\infty}\left(G_{1}^{\prime}\left(x_{1 n}\right) \otimes G_{2}^{\prime}\left(x_{2 n}\right)\right)=0$ and then that

$$
\begin{aligned}
\left(\alpha_{1} \otimes \alpha_{2}\right)\left(\sum_{n=1}^{\infty}\left(G_{1}^{\prime}\left(x_{1 n}\right) \otimes G_{2}^{\prime}\left(x_{2 n}\right)\right)\right) & =\sum_{n=1}^{\infty}\left(\alpha_{1} G_{1}^{\prime}\left(x_{1 n}\right) \otimes \alpha_{2} G_{2}^{\prime}\left(x_{2 n}\right)\right) \\
& =\sum_{n=1}^{\infty}\left(L F_{1}\left(x_{1 n}\right) \otimes F_{2}\left(x_{2 n}\right)\right)=0 .
\end{aligned}
$$

Remembering that $\alpha_{1}$ and $\alpha_{2}$ are automorphisms and applying $T$ we find $\sum_{n=1}^{\infty} L F_{1}\left(x_{1 n}\right) F_{2}\left(x_{2 n}\right) \equiv L_{3}\left(\sum_{n=1}^{\infty} F_{1}\left(x_{1 n}\right) F_{2}\left(x_{2 n}\right)\right)=L_{3} F_{3}(z)=0$ (where $L_{3}: A_{3} / M_{3} \rightarrow A_{3} / M_{3}^{\prime}$ is defined by the initial identity). Since $L_{3}\left(F_{1}\left(u_{1}\right) F_{2}\left(u_{2}\right)\right) \neq 0$ and since $A_{3} / M_{3}$ is simple we see $F_{3}(z)=0$, and so $M_{3}^{\prime} \subset M_{3}$.

Case 3. Both $N_{1}$ and $N_{2}$ are not maximal. Argue mutatis mutandis as in Case 2.

Case II. Not both $A_{1}$ and $A_{2}$ have identities. Let $M_{4}$ be a regular maximal ideal in $A_{4}$ such that (Lemma 4) $A_{4}^{0} D M_{4} \supset M_{3}$. Let $M_{4}$ engender $M_{i_{i}}$, regular maximal ideals of $A_{i e_{i}}, i=1,2$ (Case I). If $M_{10_{1}}$, say, is $A_{1}$ then clearly $A_{3} / M_{4}=0$, whence $u / M_{4}=0$, a contradiction. Hence $M_{i \epsilon_{i}} \nsubseteq A_{i}$, and the regular maximal ideals we seek are $M_{i} \equiv M_{i e_{i}} \cap A_{i}, i=1,2$.

In every case we get a pair $\left(M_{1}, M_{2}\right)$ in $\mathfrak{M}_{1} \times \mathfrak{M}_{2}$ and this pair in turn engenders $M_{3}$. Since the association $\mathfrak{M}_{1} \times \mathfrak{M}_{2} \rightarrow \mathfrak{M}_{3}$ is 1-1, $\left(M_{1}, M_{2}\right)$ is uniquely determined by $M_{3}:\left(M_{1}, M_{2}\right)=\bar{t}\left(M_{3}\right)$. We have shown $\bar{t}$ is $1-1$ and that $\bar{t}\left(\mathfrak{M}_{3}\right)=\mathfrak{M}_{1} \times \mathfrak{M}_{2}$. 
THEOREM. When hk-topologies are used throughout, $\bar{t}$ is continuous but not generally bicontinuous.

Proof. We shall show that $\bar{t}^{-1}$ is closed. Let $F_{1}$ be closed in $\mathfrak{M}_{1}$. Then $F_{3}=F_{1} \times \mathfrak{M}_{2}$ is closed in $\mathfrak{M}_{1} \times \mathfrak{M}_{2}$. Let $K_{3}=\bar{t}^{-1}\left(F_{3}\right)$. We show that $K_{3}$ is closed. To this end let $M_{3}^{0} \supset k\left(K_{3}\right)$ and assume $M_{3}^{0} \notin K_{3}$. Thus if $\bar{t}\left(M_{3}^{0}\right)=\left(M_{1}^{0}, M_{2}^{0}\right)$, then $M_{1}^{0} \notin F_{1}$. Hence $M_{1}^{0} D k\left(F_{1}\right)$ and there is an $x$ in $k\left(F_{1}\right)$ such that $x / M_{1}^{0} \neq 0$. But if $y$ is an arbitrary element of $A_{2}$, then $z=x \otimes y$ in $A_{3}$ is actually in $k\left(K_{3}\right)$. For if $M_{3}=\bar{t}^{-1}\left(M_{1}, M_{2}\right)$ is in $K_{3}$, then $M_{1}$ is in $F_{1}$ and hence $x / M_{1}=0$, whence $z / M_{3}$ $=\left(x / M_{1}\right) \otimes\left(y / M_{2}\right)=0$. Therefore $z$ is in $M_{3}$, and since $M_{3}$ is arbitrary in $K_{3}, z$ is in $k\left(K_{3}\right)$. Thus any such $z$ is in $M_{3}^{0}$. But if we choose $y$ so that $y / M_{2}^{0} \neq 0$, then $z / M_{3}^{0} \neq 0$. This contradiction shows that $K_{3}$ is closed. Similarly, if $F_{2}$ is closed in $\mathfrak{M}_{2}$ then $\bar{t}^{-1}\left(\mathfrak{M}_{1} \times F_{2}\right)$ is closed.

Since any closed set in $\mathfrak{M}_{1} \times \mathfrak{M}_{2}$ is of the form

$$
\bigcap_{r}\left[\left(F_{1}^{r} \times \mathfrak{M}_{2}\right) \cup\left(\mathfrak{M R}_{1} \times F_{2}^{r}\right)\right]
$$

where $F_{i}^{r}$ is closed in $\mathfrak{M}_{i}, i=1,2$, it follows that $\bar{t}^{-1}$ is closed and thus that $\bar{t}$ is continuous.

We are indebted to John Lindberg for suggesting the following example showing that $\bar{t}^{-1}$ need not be continuous.

EXAMPLE. Let $A_{1}$ be the commutative Banach algebra of functions analytic in the interior of the unit disc $D_{z}$ and continuous on the entire unit disc $D_{z}$. The maximal ideal space of $A_{1}$ may be identified with $D_{z}$ although the $h k$-topology constitutes a genuine weakening of the usual topology of $D_{z}$. In fact, a $h k$-closed set $F$ in $D_{z}$ has only countably many points in the (usual) interior of $D_{z}$ unless $F$ is the whole of $D_{z}$. Let $A_{2}$ be $C\left(D_{w}\right)$, i.e., the algebra of all continuous functions on $D_{w}$. Then the maximal ideal space of $A_{2}$ in the $h k$-topology is homeomorphic with $D_{w}$ in its usual topology.

In $A_{3}$ let $v$ be the element $z \otimes 1-1 \otimes w(z$ and $w$ here are the usual complex variables). Then in $\mathfrak{M}_{1} \times \mathfrak{M}_{2}$ the set $K=\{(z, w) \mid z=w\}$ is the hull of $v$, and so $\bar{t}^{-1}(K)$ is closed in the $h k$-topology of $\mathfrak{M}_{3}$. Thus $U=\mathfrak{M}_{3} \backslash \bar{t}^{-1}(K)$ is open. We shall show that $\bar{t}(U)$ is not open in $\mathfrak{M}_{1} \times \mathfrak{M}_{2}$.

If $\bar{t}(U)$ were open, there would be open sets $U_{i}$ in $\mathfrak{M}_{i}$ such that $U_{1} \times U_{2} \subset \bar{t}(U)$, and $U_{2}$ could be taken as an ordinary open circle in the interior of $D_{w}$. Since $F_{1}=\mathfrak{M}_{1} \backslash U_{1}$ is $h k$-closed in $D_{z}, F_{1}$ has at most countably many points in the ordinary interior of $D_{z}$. Let $U_{2}^{*}$ be the circle in $D_{z}$ that consists of the same set of complex numbers as the set comprising $U_{2}$. Then $U_{2}^{*} \backslash F_{1} \neq \varnothing$ since $F_{1}$ meets the ordinary interior of $D_{z}$ in at most countably many points. But then $U_{1} \times U_{2}$ $\supset\left(U_{2}^{*} \backslash F_{1}\right) \times U_{2}$. Clearly, any point $z$ in $U_{2}^{*} \backslash F_{1}$ corresponds to a point 
$w$ in $U_{2}$ such that $z=w$, i.e., $\left(U_{2}^{*} \backslash F_{1}\right) \times U_{2}$ meets $K$ and thus $U_{1} \times U_{2}$ cannot lie in the complement $\bar{t}(U)$ of $K$.

REMARK. In the last paragraph of p. 302 and the first two lines of p. 303 [2] the proof given is incorrect since the $u_{i}$ chosen there vary with the $M_{i}$. The following amended proof should be substituted.

Let $M_{03}$ be in $\mathfrak{M}_{3}$ and if $\bar{t}\left(M_{03}\right)=\left(M_{01}, M_{02}\right)$, let $N\left(M_{0 i}\right)$ in $\mathfrak{M}_{i}$ be of the form:

$$
\begin{array}{r}
N\left(M_{0 i}\right)=\left\{M_{i}|| a_{j i}^{+}\left(M_{i}\right)-a_{j i}^{+}\left(M_{0 i}\right) \mid<r_{i}, j=1,2, \cdots, J_{i}\right\}, \\
i=1,2 .
\end{array}
$$

To prove the continuity of $\bar{t}$ it suffices to find an $N\left(M_{03}\right)$ such that $\bar{t}\left(N\left(M_{03}\right)\right) \subset N\left(M_{01}\right) \times N\left(M_{02}\right)$. To this end let $u_{0 i}$ be identities modulo $M_{0 i}, i=1,2$ and let

$$
\begin{gathered}
N\left(M_{03}\right)=\left\{M_{3}||\left(a_{j 1}^{k} \otimes u_{02}\right)^{+}\left(M_{3}\right)-\left(a_{j 1}^{k} \otimes u_{02}\right)^{+}\left(M_{03}\right) \mid<s_{k},\right. \\
k=1,2, j=1,2, \cdots, J_{1} ; \\
\left|\left(u_{01} \otimes a_{j 2}^{m}\right)^{+}\left(M_{3}\right)-\left(u_{01} \otimes a_{j 2}^{m}\right)^{+}\left(M_{03}\right)\right|<v_{m}, \\
m=1,2, j=1,2, \cdots, J_{2} ; \\
\left.\left|\left(u_{01} \otimes u_{02}\right)^{+}\left(M_{3}\right)-1\right|<w\right\} .
\end{gathered}
$$

We treat two cases: (1) For all $(j, i) a_{j i}^{+}\left(M_{0 i}\right) \neq 0$. Then for appropriate choices of the $s_{k}, v_{m}$ and $w$, we can assure that

$$
\left|\frac{\left(a_{j 1}^{2} \otimes u_{02}\right)^{+}\left(M_{03}\right)}{\left(a_{j 1} \otimes u_{02}\right)^{+}\left(M_{03}\right)}-\frac{\left(a_{j 1}^{2} \otimes u_{02}\right)^{+}\left(M_{3}\right)}{\left(a_{j 1} \otimes u_{02}\right)^{+}\left(M_{3}\right)}\right| \equiv\left|a_{j 1}^{+}\left(M_{01}\right)-a_{j 1}^{+}\left(M_{1}\right)\right|<r_{1}
$$

and similarly that $\left|a_{j 2}^{+}\left(M_{02}\right)-a_{j 2}^{+}\left(M_{2}\right)\right|<r_{2}$, where $\bar{t}\left(M_{3}\right)=\left(M_{1}, M_{2}\right)$. In this case then, $\bar{t}\left(N\left(M_{03}\right)\right) \subset N\left(M_{01}\right) \times N\left(M_{02}\right)$. (2) Some $a_{\mathfrak{H}}^{+}\left(M_{0 i}\right)$ $=0$. For the $(j, i)$ combinations for which $a_{\mathfrak{j}}^{+}\left(M_{0 i}\right) \neq 0, s_{k}, v_{m}$ and $w$ are chosen as in (1). For the other $(j, i)$ combinations, since $\left|u_{0 i}^{+}\left(M_{i}\right)\right|$ is bounded, we see that for suitable $w$ and $M_{3}$ in $N\left(M_{03}\right)\left|u_{0 i}^{+}\left(M_{i}\right)\right|$ is bounded away from 0 and then for suitable $s_{k}$ and $v_{m}\left|a_{\mathfrak{f}}^{+}\left(M_{i}\right)\right|$ is small. The result follows upon a final revised choice of $s_{k}, v_{m}$ and $w$.

\section{BiBLIOGRAPHY}

1. N. Bourbaki, Algèbre, Chapter III, Paris, 1948.

2. B. Gelbaum, Tensor products of Banach algebras, Canad. J. Math. vol. 11 (1959) pp. 297-310.

3. A. Grothendieck, Produits tensoriels topologiques et espaces nucleaires, Memoirs Amer. Math. Soc. no. 16, 1955.

4. R. Schatten, A theory of cross-spaces, Princeton, New Jersey, Princeton University Press, 1950.

UNIVERSITY OF MINNESOTA 